\title{
Convergence of the Euler Method of Stochastic Differential Equations with Piecewise Continuous Arguments
}

\author{
Ling Zhang, ${ }^{1,2}$ and Minghui Song ${ }^{1}$ \\ ${ }^{1}$ Department of Mathematics, Harbin Institute of Technology, Harbin 150001, China \\ 2 Institute of Mathematical Sciences, Daqing Normal University, Daqing 163712, China \\ Correspondence should be addressed to Minghui Song, songmh@1sec.cc.ac.cn
}

Received 20 June 2012; Accepted 29 October 2012

Academic Editor: Roman Dwilewicz

Copyright (C) 2012 L. Zhang and M. Song. This is an open access article distributed under the Creative Commons Attribution License, which permits unrestricted use, distribution, and reproduction in any medium, provided the original work is properly cited.

\begin{abstract}
The main purpose of this paper is to investigate the strong convergence of the Euler method to stochastic differential equations with piecewise continuous arguments (SEPCAs). Firstly, it is proved that the Euler approximation solution converges to the analytic solution under local Lipschitz condition and the bounded $p$ th moment condition. Secondly, the Euler approximation solution converge to the analytic solution is given under local Lipschitz condition and the linear growth condition. Then an example is provided to show which is satisfied with the monotone condition without the linear growth condition. Finally, the convergence of numerical solutions to SEPCAs under local Lipschitz condition and the monotone condition is established.
\end{abstract}

\section{Introduction}

Recently, differential equations with piecewise continuous arguments (EPCAs) have attracted much attention, and many useful conclusions have been obtained. These systems have applications in certain biomedical models, control systems with feedback delay in the work of Cooke and Wiener [1]. The general theory and basic results for EPCAs have by now been thoroughly investigated in the book of Wiener [2]. Song et al. [3] deal with the stability analysis of numerical methods for the solution of advanced differential equations with piecewise continuous arguments. A typical EPCA contains arguments that are constant on certain intervals. The solutions are determined by a finite set of initial data, rather than by an initial function, as in the case of general functional differential equation. A solution 
is defined as a continuous, sectionally smooth function that satisfies the equation within these intervals. Continuity of a solution at a point joining any two consecutive intervals leads to recursion relations for the solution at such points. Hence, EPCAs represent a hybrid of continuous and discrete dynamical systems and combine the properties of both differential and difference equations.

However, up to now there are few people who have considered the influence of noise to EPCAs. Actually, the environment and accidental events may greatly influence the systems. Thus analyzing SEPCAs is an interesting topic both in theory and applications. There is in general no explicit solution to an SEPCA, hence numerical solutions are required in practice. Numerical solutions to stochastic differential equations (SDEs) have been discussed under the local Lipschitz condition and the linear growth condition by many authors (see $[4,5])$. Mao [6] discusses numerical solutions to stochastic differential delay equations (SDDEs) under the local Lipschitz condition and the linear growth condition. Mao and Sabanis [7] discuss numerical solutions to SDDEs with variable delay under the local Lipschitz condition and the linear growth condition. Mao discusses numerical solutions to SDEs and SDDEs under the local Lipschitz condition and the monotone condition (see [8]). Dai and Liu [9] give the mean-square stability of the numerical solutions of linear SEPCAs. However, SEPCAs do not have the convergence results. The main aim of this paper is to establish convergence of numerical solution for SEPCAs under the differential conditions.

The paper is organized as follows. In Section 2, we introduce necessary notations and the Euler method. In Section 3, the strong convergence of the Euler-Maruyama method to SEPCAs under local Lipschitz condition and the bounded $p$ th moment condition will be given. In Section 4, the strong convergence of the Euler-Maruyama method to SEPCAs under local Lipschitz condition and the linear growth condition will be presented. In Section 5, an example is provided to show which is satisfied with the monotone condition without the linear growth condition. In Section 6, we obtain the convergence of numerical solutions to SEPCAs under local Lipschitz condition and the monotone condition is established.

\section{Preliminary Notation and Euler Method}

In this paper, unless otherwise specified, let $|x|$ be the Euclidean norm in $x \in R^{n}$. If $A$ is a vector or matrix, its transpose is defined by $A^{T}$. If $A$ is a matrix, its trace norm is defined by $|A|=\sqrt{\operatorname{trace}\left(A^{T} A\right)}$. For simplicity, we also have to denote by $a \wedge b=\min \{a, b\}, a \vee b=$ $\max \{a, b\}$.

Let $(\Omega, \mathcal{F}, P)$ be a complete probability space with a filtration $\left\{\mathcal{F}_{t}\right\}_{t>0}$, satisfying the usual conditions. $\mathcal{L}^{1}\left([0, \infty), R^{n}\right)$ and $\mathcal{L}^{2}\left([0, \infty), R^{n}\right)$ denote the family of all real valued $\mathcal{F}_{t^{-}}$ adapted process $f(t)_{t \geq 0}$, such that for every $T>0, \int_{0}^{T}|f(t)| d t<\infty$ a.s. and $\int_{0}^{T}|f(t)|^{2} d t<$ $\infty$ a.s., respectively. For any $a, b \in R$ with $a<b$, denote $C\left([a, b] ; R^{n}\right)$ the family of continuous functions $\phi$ from $[a, b]$ to $R^{n}$ with the norm $\|\phi\|=\sup _{a \leq \theta \leq b}|\phi(\theta)|$. Denote $C_{q_{t}}^{b}\left([a, b] ; R^{n}\right)$ the family of all bounded $\mathcal{F}_{t}$-measurable $C\left([a, b] ; R^{n}\right)$-valued random variables. Let $B(t)=$ $\left(B_{1}(t), \ldots, B_{d}(t)\right)^{T}$ be a $d$-dimensional Brownian motion defined on the probability space.

Throughout this paper, we consider stochastic differential equations with piecewise continuous arguments:

$$
d x(t)=f(x(t), x([t])) d t+g(x(t), x([t])) d B(t) \quad \forall t \geq 0,
$$


with initial data $x(0)=x_{0}$, where $f: R^{n} \times R^{n} \rightarrow R^{n}, g: R^{n} \times R^{n} \rightarrow R^{n \times d}, x_{0}$ is a vector, and [·] denotes the greatest-integer function. By the definition of stochastic differential, this equation is equivalent to the following stochastic integral equation:

$$
x(t)=x(0)+\int_{0}^{t} f(x(s), x([s])) d s+\int_{0}^{t} g(x(s), x([s])) d B(s) \quad \forall t \geq 0 .
$$

Moreover, we also require the coefficients $f$ and $g$ to be sufficiently smooth.

To be precise, let us state the following conditions.

(H1) The local Lipschitz condition: for every integer $i \geq 1$, there exists a positive constant $L_{i}$ such that

$$
|f(x, y)-f(\bar{x}, \bar{y})|^{2} \vee|g(x, y)-g(\bar{x}, \bar{y})|^{2} \leq L_{i}\left(|x-\bar{x}|^{2}+|y-\bar{y}|^{2}\right)
$$

for those $x, \bar{x}, y, \bar{y} \in R^{n}$ with $|x| \vee|\bar{x}| \vee|y| \vee|\bar{y}| \leq i$.

(H2) Linear growth condition: there exists a positive constant $K$ such that

$$
|f(x, y)|^{2} \vee|g(x, y)|^{2} \leq K\left(1+|x|^{2}+|y|^{2}\right),
$$

for all $(x, y) \in R^{n} \times R^{n}$.

(H3) Monotone condition: there exists a positive constant $K_{1}$ such that

$$
x^{T} f(x, y)+\frac{p-1}{2}|g(x, y)|^{2} \leq K_{1}\left(1+|x|^{2}+|y|^{2}\right) \text {, }
$$

for all $(x, y) \in R^{n} \times R^{n}$.

(H4) The bounded $p$ th moment condition: there exists a pair of constants $p>2$ and $K_{2}>0$ such that

$$
E\left[\sup _{0 \leq t \leq T}|x(t)|^{p}\right] \vee E\left[\sup _{0 \leq t \leq T}|y(t)|^{p}\right] \leq K_{2} .
$$

Let us first give the definition of the solution.

Definition 2.1 (see [10]). An $R^{n}$-valued stochastic process $\{x(t)\}$ is called a solution of (2.1) on $[0, \infty)$, if it has the following properties:

(1) $\{x(t)\}$ is continuous on $[0, \infty)$ and $\mathcal{F}_{t}$ adapted;

(2) $\{f(x(t), x([t]))\} \in \mathcal{L}^{1}\left([0, \infty), R^{n}\right)$ and $\{g(x(t), x([t]))\} \in \mathcal{L}^{2}\left([0, \infty), R^{n \times d}\right)$;

(3) Equation (2.2) is satisfied on each interval $[n, n+1) \subset[0, \infty)$ with integral end points almost surely. A solution $\{x(t)\}$ is said to be unique if any other solution $\{\bar{x}(t)\}$ is indistinguishable from $\{x(t)\}$, that is,

$$
P\{x(t)=\bar{x}(t) \forall t \in[0, \infty)\}=1 .
$$


Let $h=1 / m$ be a given stepsize with integer $m \geq 1$ and the grid points $t_{n}$ defined by $t_{n}=$ $n h(n=0,1,2, \ldots)$. For simplicity, we assume $T=N h$. We consider the Euler-Maruyama method to (2.1),

$$
y_{n+1}=y_{n}+f\left(y_{n}, y^{h}([n h])\right) h+g\left(y_{n}, y^{h}([n h])\right) \Delta B_{n}
$$

for $n=0,1,2, \ldots$, where $\Delta B_{n}=B\left(t_{n}\right)-B\left(t_{n-1}\right), y^{h}([n h])$ is approximation to the exact solution $x([n h])$. Let $n=k m+l(k=0,1,2, \ldots, l=0,1,2, \ldots, m-1)$. The adaptation of the Euler method to (2.1) leads to a numerical process of the following type:

$$
y_{k m+l+1}=y_{k m+l}+f\left(y_{k m+l}, y_{k m}\right) h+g\left(y_{k m+l}, y_{k m}\right) \Delta B_{k m+l},
$$

where $\Delta B_{k m+l}=B\left(t_{k m+l}\right)-B\left(t_{k m+l-1}\right), y_{k m+l}$ and $y_{k m}$ are approximations to the exact solution $x\left(t_{k m+l}\right)$ and $x\left(\left[t_{k m+l}\right]\right)$, respectively. The continuous Euler-Maruyama approximate solution is defined by

$$
y(t)=y(0)+\int_{0}^{t} f(z(s), z([s])) d s+\int_{0}^{t} g(z(s), z([s])) d B(s),
$$

where $z(t)=y_{k m+l}$ and $z([t])=y_{k m}$ for $t \in\left[t_{k m+l}, t_{k m+l+1}\right)$. It is not difficult to see that $y\left(t_{k m+l}\right)=z\left(t_{k m+l}\right)=y_{k m+l}$ for $k=0,1,2, \ldots, l=0,1,2, \ldots, m-1$. For sufficiently large integer $i$, define the stopping times $\eta_{i}=\inf \{t \geq 0:|x(t)| \geq i\}, \theta_{i}=\inf \{t \geq 0:|y(t)| \geq i\}, \tau_{i}=\eta_{i} \wedge \theta_{i}$.

\section{Convergence of the Euler-Maruyama Method under the Bounded pth Moment}

We will show the strong convergence of the EM method on (2.1) under local Lipschitz condition and the bounded $p$ th moment condition. The following lemma shows that both $y(t)$ and $z(t)$ are close to each other.

Lemma 3.1. Under the condition (H1), let $T>0$ be arbitrary. Then

$$
E \sup _{0 \leq t \leq T}|y(t)-z(t)|^{2} \leq C_{1}\left(x_{0}, i\right) h
$$

where $C_{1}\left(x_{0}, i\right)=4 L_{i}(T+4) K_{2}$. 
Proof. For $t \in[0, T)$, there are two integers $k$ and $l$ such that $t \in\left[t_{k m+l}, t_{k m+l+1}\right)$. By the Hölder inequality, we compute

$$
\begin{aligned}
|y(t)-z(t)|^{2} & =\left|\int_{t_{k m+l}}^{t} f(z(s), z([s])) d s+\int_{t_{k m+l}}^{t} g(z(s), z([s])) d B(s)\right|^{2} \\
& \leq 2\left|\int_{t_{k m+l}}^{t} f(z(s), z([s])) d s\right|^{2}+2\left|\int_{t_{k m+l}}^{t} g(z(s), z([s])) d B(s)\right|^{2} \\
& \leq 2 T \int_{t_{k m+l}}^{t}|f(z(s), z([s]))|^{2} d s+2\left|\int_{t_{k m+l}}^{t} g(z(s), z([s])) d B(s)\right|^{2} .
\end{aligned}
$$

This implies that, for any $0 \leq t_{1} \leq T$,

$$
\begin{aligned}
E \sup _{0 \leq t \leq t_{1}}|y(t)-z(t)|^{2} \leq & 2 T E \sup _{0 \leq t \leq t_{1}} \int_{t_{k m+l}}^{t}|f(z(s), z([s]))|^{2} d s \\
& +2 E \sup _{0 \leq t \leq t_{1}}\left|\int_{t_{k m+l}}^{t} g(z(s), z([s])) d B(s)\right|^{2} .
\end{aligned}
$$

By the Doob martingale inequality, we have

$$
E \sup _{0 \leq t \leq t_{1}}|y(t)-z(t)|^{2} \leq 2 T E \int_{t_{k m+l}}^{t_{1}}|f(z(s), z([s]))|^{2} d s+8 E \int_{t_{k m+l}}^{t_{1}}|g(z(s), z([s]))|^{2} d s .
$$

Using the local Lipschitz conditions

$$
\begin{aligned}
E \sup _{0 \leq t \leq t_{1}}|y(t)-z(t)|^{2} & \leq 2 L_{i}(T+4) E \int_{t_{k m+l}}^{t_{1}}\left(|z(s)|^{2}+|z([s])|^{2}\right) d s \\
& \leq 4 L_{i}(T+4) \int_{t_{k m+l}}^{t_{1}}\left(E \sup _{0 \leq u \leq s}|y(u)|^{2}\right) d s \\
& \leq C_{1}\left(x_{0}, i\right) h,
\end{aligned}
$$

where $C_{1}\left(x_{0}, i\right)=4 L_{i}(T+4) K_{2}$. The proof is completed.

Theorem 3.2. Under the conditions (H1) and (H4), the EM approximate solution converges to the exact solution of (2.1) in the sense that

$$
\lim _{h \rightarrow 0} E\left[\sup _{0 \leq t \leq T}|y(t)-x(t)|^{2}\right]=0 .
$$


Proof. Fix a $p>2$; let $e(t)=x(t)-y(t)$; it is easy to see that

$$
\begin{aligned}
E\left[\sup _{0 \leq t \leq T}|e(t)|^{2}\right] & =E\left[\sup _{0 \leq t \leq T}|e(t)|^{2} 1_{\left\{\theta_{i}>T\right.} \text { and } \eta_{i}>T\right\} \\
& \\
& =E\left[\sup _{0 \leq t \leq T}|e(t)|^{2} 1_{\left\{\tau_{i}>T\right\}}\right]+E\left[\sup _{0 \leq t \leq T}|e(t)|^{2} 1_{\left\{\theta_{i} \leq T \text { or } \eta_{i} \leq T\right\}}|e(t)|^{2} 1_{\left\{\theta_{i} \leq T \text { or } \eta_{i} \leq T\right\}}\right] \\
& \leq E\left[\sup _{0 \leq t \leq T}\left|e\left(t \wedge \tau_{i}\right)\right|^{2}\right]+E\left[\sup _{0 \leq t \leq T}|e(t)|^{2} 1_{\left\{\theta_{i} \leq T \text { or } \eta_{i} \leq T\right\}}\right] .
\end{aligned}
$$

By the Young inequality $x y \leq\left(x^{p} / p\right)+\left(y^{q} / q\right)$, for any $a, b, p, q, \delta>0,(1 / p)+(1 / q)=1$, we have

$$
a b \leq a \delta^{1 / p} \frac{b}{\delta^{1 / p}} \leq \frac{\left(a \delta^{1 / p}\right)^{p}}{p}+\frac{b^{q}}{q \delta^{q / p}}=\frac{a^{p} \delta}{p}+\frac{b^{q}}{q \delta^{q / p}}
$$

Thus for any $\delta>0$, we have

$$
E\left[\sup _{0 \leq t \leq T}|e(t)|^{2} 1_{\left\{\theta_{i} \leq T \text { or } \eta_{i} \leq T\right\}}\right] \leq \frac{2 \delta}{p} E\left[\sup _{0 \leq t \leq T}|e(t)|^{p}\right]+\frac{1-(2 / p)}{\delta^{2 /(p-2)}} P\left\{\theta_{i} \leq T \text { or } \eta_{i} \leq T\right\}
$$

By condition (H4), we have

$$
P\left(\theta_{i} \leq T\right)=E\left[1_{\left\{\theta_{i} \leq T\right\}} \frac{\left|y\left(\theta_{i}\right)\right|^{p}}{i^{p}}\right] \leq \frac{1}{i^{p}} E\left[\sup _{0 \leq t \leq T}|y(t)|^{p}\right] \leq \frac{K_{2}}{i^{p}}
$$

Similarly, the result is

$$
P\left(\eta_{i} \leq T\right) \leq \frac{K_{2}}{i^{p}} .
$$

So that

$$
P\left(\theta_{i} \leq T \text { or } \eta_{i} \leq T\right) \leq P\left(\theta_{i} \leq T\right)+P\left(\eta_{i} \leq T\right) \leq \frac{2 K_{2}}{i^{p}}
$$

Using these bounds, then

$$
\begin{aligned}
& E\left[\sup _{0 \leq t \leq T}|e(t)|^{p}\right] \leq 2^{p-1}\left(E\left[\sup _{0 \leq t \leq T}|x(t)|^{p}\right]+E\left[\sup _{0 \leq t \leq T}|y(t)|^{p}\right]\right) \\
& \leq 2^{p} K_{2} . \\
& E\left[\sup _{0 \leq t \leq T}|e(t)|^{2} 1_{\left\{\theta_{i} \leq T \text { or } \eta_{i} \leq T\right\}}\right] \leq \frac{2^{p+1} \delta K_{2}}{p}+\frac{2(p-2) K_{2}}{p \delta^{2 /(p-2)} i^{p}} .
\end{aligned}
$$


By the definitions of $x(t)$ and $y(t)$, we have

$$
\begin{aligned}
\left|x\left(t \wedge \tau_{i}\right)-y\left(t \wedge \tau_{i}\right)\right|^{2} \leq & 2 \mid \int_{0}^{t \wedge \tau_{i}}\left[\left.f(x(s), x([s])-f(z(s), z([s]))] d s\right|^{2}\right. \\
& +2 \mid \int_{0}^{t \wedge \tau_{i}}\left[\left.g(x(s), x([s])-g(z(s), z([s]))] d B(s)\right|^{2} .\right.
\end{aligned}
$$

Thus, for any $t_{1} \in[0, T]$

$$
\begin{aligned}
E \sup _{0 \leq t \leq t_{1}}\left|x\left(t \wedge \tau_{i}\right)-y\left(t \wedge \tau_{i}\right)\right|^{2} \leq & 2 E \sup _{0 \leq t \leq t_{1}} \mid \int_{0}^{t \wedge \tau_{i}}\left[\left.f(x(s), x([s])-f(z(s), z([s]))] d s\right|^{2}\right. \\
& +2 E \sup _{0 \leq t \leq t_{1}} \mid \int_{0}^{t \wedge \tau_{i}}\left[\left.g(x(s), x([s])-g(z(s), z([s]))] d B(s)\right|^{2} .\right.
\end{aligned}
$$

By the Hölder inequality, condition (H1), and Lemma 3.1, one gets

$$
\begin{aligned}
E \sup _{0 \leq t \leq t_{1}} \mid \int_{0}^{t \wedge \tau_{i}}\left[\left.f(x(s), x([s])-f(z(s), z([s]))] d s\right|^{2}\right. \\
\leq T E \int_{0}^{t_{1} \wedge \tau_{i}} \mid f\left(x(s), x([s])-\left.f(z(s), z([s]))\right|^{2} d s\right. \\
=T E \int_{0}^{t_{1}} \mid f\left(x\left(s \wedge \tau_{i}\right), x\left(\left[s \wedge \tau_{i}\right]\right)-\left.f\left(z\left(s \wedge \tau_{i}\right), z\left(\left[s \wedge \tau_{i}\right]\right)\right)\right|^{2} d s\right. \\
\leq T L_{i} E \int_{0}^{t_{1}}\left(\left|x\left(s \wedge \tau_{i}\right)-z\left(s \wedge \tau_{i}\right)\right|^{2}+\left|x\left(\left[s \wedge \tau_{i}\right]\right)-z\left(\left[s \wedge \tau_{i}\right]\right)\right|^{2}\right) d s \\
\leq 2 T L_{i} \int_{0}^{t_{1}}\left(E\left|x\left(s \wedge \tau_{i}\right)-y\left(s \wedge \tau_{i}\right)\right|^{2}+E\left|x\left(\left[s \wedge \tau_{i}\right]\right)-y\left(\left[s \wedge \tau_{i}\right]\right)\right|^{2}\right) d s \\
\quad+2 T L_{i} \int_{0}^{t_{1}}\left(E\left|y\left(s \wedge \tau_{i}\right)-z\left(s \wedge \tau_{i}\right)\right|^{2}+E\left|y\left(\left[s \wedge \tau_{i}\right]\right)-z\left(\left[s \wedge \tau_{i}\right]\right)\right|^{2}\right) d s \\
\leq 2 T L_{i} \int_{0}^{t_{1}}\left(E\left|x\left(s \wedge \tau_{i}\right)-y\left(s \wedge \tau_{i}\right)\right|^{2}+E\left|x\left(\left[s \wedge \tau_{i}\right]\right)-y\left(\left[s \wedge \tau_{i}\right]\right)\right|^{2}\right) d s+4 T^{2} L_{i} C_{1}\left(x_{0}, i\right) h \\
\leq 4 T L_{i} \int_{0}^{t_{1}} E \sup _{0 \leq v \leq s \wedge \tau_{i}}|x(v)-y(v)|^{2} d s+4 T^{2} L_{i} C_{1}\left(x_{0}, i\right) h .
\end{aligned}
$$


Similarly, by the Burkhôlder-Davis-Gundy inequality, then

$$
\begin{aligned}
& E \sup _{0 \leq t \leq t_{1}}\left|\int_{0}^{t \wedge \tau_{i}}[g(x(s), x([s]))-g(z(s), z([s]))] d B(s)\right|^{2} \\
& \quad \leq 4 E \int_{0}^{t \wedge \tau_{i}}|g(x(s), x([s]))-g(z(s), z([s]))|^{2} d s \\
& \quad \leq 16 L_{i} \int_{0}^{t_{1}} E \sup _{0 \leq v \leq s \wedge \tau_{i}}|x(v)-y(v)|^{2} d s+16 T L_{i} C_{1}\left(x_{0}, i\right) h .
\end{aligned}
$$

Substituting (3.16) and (3.17) into (3.15) gives

$$
E \sup _{0 \leq t \leq t_{1}}\left|x\left(t \wedge \tau_{i}\right)-y\left(t \wedge \tau_{i}\right)\right|^{2} \leq 8(T+4) L_{i} \int_{0}^{t_{1}} E \sup _{0 \leq v \leq s \wedge \tau_{i}}|x(v)-y(v)|^{2} d s+8 T(T+4) L_{i} C_{1}\left(x_{0}, i\right) h .
$$

By the Gronwall inequality, we must get

$$
E \sup _{0 \leq t \leq T}\left|x\left(t \wedge \tau_{i}\right)-y\left(t \wedge \tau_{i}\right)\right|^{2} \leq C_{2}\left(x_{0}, i\right) h
$$

where $C_{2}\left(x_{0}, i\right)=8 T(T+4) L_{i} C_{1}\left(x_{0}, i\right) e^{8 T(T+4) L_{i}}$. So we have

$$
\begin{aligned}
E\left[\sup _{0 \leq t \leq T}|e(t)|^{2}\right] & \leq E\left[\sup _{0 \leq t \leq T}\left|e\left(t \wedge \tau_{i}\right)\right|^{2}\right]+E\left[\sup _{0 \leq t \leq T}|e(t)|^{2} 1_{\left\{\theta_{i}<T \text { or } \eta_{i}<T\right\}}\right] \\
& \leq C_{2}\left(x_{0}, i\right) h+\frac{2^{p+1} \delta K_{2}}{p}+\frac{2(p-2) K_{2}}{p \delta^{2 /(p-2)} i^{p}} .
\end{aligned}
$$

Given any $\epsilon>0$, we can choose $\delta$ sufficiently small for

$$
\frac{2^{p+1} \delta K_{2}}{p}<\frac{\epsilon}{3}
$$

then choose $i$ sufficiently large for

$$
\frac{2(p-2) K_{2}}{p \delta^{2 /(p-2)} i^{p}}<\frac{\epsilon}{3}
$$


and finally choose $h$ sufficiently small, so that

$$
C_{2}\left(x_{0}, i\right) h<\frac{\epsilon}{3}
$$

Thus $E\left[\sup _{0 \leq t \leq T}|e(t)|^{2}\right]<\epsilon$. The proof is completed.

\section{Convergence of the Euler-Maruyama Method under Linear Growth Condition}

We will show the strong convergence of the EM method on (2.1) under local Lipschitz condition and the linear growth condition. In the following we will show that the linear growth condition (H2) implies the bounded $p$ th moment condition (H4).

Lemma 4.1. Under the linear growth conditions (H2), there exists a positive constant $C_{3}$ such that the solution of (2.1) satisfies

$$
E \sup _{0 \leq t \leq T}|x(t)|^{p} \leq C_{3}\left(1+\left|x_{0}\right|^{p}\right)
$$

where $C_{3}=C_{3}(p, T, K)$ is a constant independent of $h$.

Proof. It follows from (2.2) that

$$
\begin{aligned}
|x(t)|^{p} & =\left|x(0)+\int_{0}^{t} f(x(s), x([s])) d s+\int_{0}^{t} g(x(s), x([s])) d B(s)\right|^{p} \\
& \leq 3^{p-1}\left[|x(0)|^{p}+\left|\int_{0}^{t} f(x(s), x([s])) d s\right|^{p}+\left|\int_{0}^{t} g(x(s), x([s])) d B(s)\right|^{p}\right] .
\end{aligned}
$$

By the Hölder inequality, we obtain

$$
|x(t)|^{p} \leq 3^{p-1}\left[|x(0)|^{p}+T^{p-1} \int_{0}^{t}|f(x(s), x([s]))|^{p} d s+\left|\int_{0}^{t} g(x(s), x([s])) d B(s)\right|^{p}\right] .
$$

This implies that, for any $0 \leq t_{1} \leq T$,

$$
\begin{gathered}
E \sup _{0 \leq t \leq t_{1}}|x(t)|^{p} \leq 3^{p-1}\left[|x(0)|^{p}+T^{p-1} E \sup _{0 \leq t \leq t_{1}} \int_{0}^{t}|f(x(s), x([s]))|^{p} d s\right. \\
\left.+E \sup _{0 \leq t \leq t_{1}}\left|\int_{0}^{t} g(x(s), x([s])) d B(s)\right|^{p}\right] .
\end{gathered}
$$


By the Burkholder-Davis-Gundy inequality and the Hölder inequality, it is not difficult to show that

$$
\begin{array}{r}
E \sup _{0 \leq t \leq t_{1}}|x(t)|^{p} \leq 3^{p-1}\left[|x(0)|^{p}+T^{p-1} E \int_{0}^{t_{1}}|f(x(s), x([s]))|^{p} d s\right. \\
\left.+C_{p} T^{p / 2-1} E \int_{0}^{t_{1}}|g(x(s), x([s]))|^{p} d s\right],
\end{array}
$$

where $C_{p}$ is a constant. Note from the linear growth conditions that

$$
\begin{aligned}
E \sup _{0 \leq t \leq t_{1}}|x(t)|^{p} \leq & 3^{p-1}\left[|x(0)|^{p}+T^{p-1} E \int_{0}^{t_{1}} K^{p / 2}\left(1+|x(s)|^{2}+|x([s])|^{2}\right)^{p / 2} d s\right. \\
& \left.+C_{p} T^{p / 2-1} E \int_{0}^{t_{1}} K^{p / 2}\left(1+|x(s)|^{2}+|x([s])|^{2}\right)^{p / 2} d s\right] \\
\leq & 3^{p-1}|x(0)|^{p}+3^{(3 p / 2)-2} K^{p / 2}\left(T^{p-1}+C_{p} T^{p / 2-1}\right) \\
& \times E \int_{0}^{t_{1}}\left(1+|x(s)|^{p}+|x([s])|^{p}\right) d s \\
\leq & 3^{p-1}|x(0)|^{p}+3^{(3 p / 2)-2} K^{p / 2}\left(T^{p}+C_{p} T^{p / 2}\right) \\
& +2 \times 3^{(3 p / 2)-2} K^{p / 2}\left(T^{p-1}+C_{p} T^{p / 2-1}\right) E \sup _{0 \leq u \leq s}|x(u)|^{p} d s .
\end{aligned}
$$

By the Gronwall inequality, we must get

$$
E \sup _{0 \leq t \leq T}|x(t)|^{p} \leq C_{3}\left(1+\left|x_{0}\right|^{p}\right)
$$

where $C_{3}=C_{3}(p, T, K)$ is a constant independent of $h$.

The following lemma shows that the continuous Euler-Maruyama approximate solution has bounded $p$ th moments.

Lemma 4.2. Under the linear growth conditions (H2), there exists a positive constant $\mathrm{C}_{4}$ such that the continuous approximate solution of the Euler-Maruyama (2.10) satisfies

$$
E \sup _{0 \leq t \leq T}|y(t)|^{p} \leq C_{4}\left(1+\left|x_{0}\right|^{p}\right)
$$

where $C_{4}=C_{4}(p, T, K)$ is a constant independent of $h$. 
Proof. By the inequality $|a+b+c|^{2} \leq 3|a|^{2}+3|b|^{2}+3|c|^{2}$ and (2.10), in the same way as Lemma 4.1, for any $0 \leq t_{1} \leq T$, we can obtain

$$
\begin{array}{r}
E \sup _{0 \leq t \leq t_{1}}|y(t)|^{p} \leq 3^{p-1}\left[|y(0)|^{p}+T^{p-1} E \int_{0}^{t_{1}}|f(z(s), z([s]))|^{p} d s\right. \\
\left.+C_{p} T^{(p / 2)-1} E \int_{0}^{t_{1}}|g(z(s), z([s]))|^{p} d s\right],
\end{array}
$$

where $C_{p}$ is a constant. Note from the linear growth conditions that

$$
\begin{aligned}
& E \sup _{0 \leq t \leq t_{1}}|y(t)|^{p} \leq 3^{p-1}\left[|y(0)|^{p}+T^{p-1} E \int_{0}^{t_{1}} K^{p / 2}\left(1+|z(s)|^{2}+|z([s])|^{2}\right)^{p / 2} d s\right. \\
& \left.+C_{p} T^{p / 2-1} E \int_{0}^{t_{1}} K^{p / 2}\left(1+|z(s)|^{2}+|z([s])|^{2}\right)^{p / 2} d s\right] \\
& \leq 3^{p-1}|y(0)|^{p}+3^{(3 p / 2)-2} K^{p / 2}\left(T^{p-1}+C_{p} T^{p / 2-1}\right) \\
& \times E \int_{0}^{t_{1}}\left(1+|z(s)|^{p}+|z([s])|^{p}\right) d s \\
& \leq 3^{p-1}|y(0)|^{p}+3^{(3 p / 2)-2} K^{p / 2}\left(T^{p}+C_{p} T^{p / 2}\right) \\
& +2 \times 3^{(3 p / 2)-2} K^{p / 2}\left(T^{p-1}+C_{p} T^{p / 2-1}\right) E \sup _{0 \leq u \leq s}|y(u)|^{p} d s .
\end{aligned}
$$

By the Gronwall inequality, we must get

$$
E \sup _{0 \leq t \leq T}|y(t)|^{p} \leq C_{4}\left(1+\left|x_{0}\right|^{p}\right)
$$

where $C_{4}=C_{4}(p, T, K)$ is a constant independent of $h$.

According to Theorem 3.2, we have the following theorem.

Theorem 4.3. Under the conditions (H1) and (H2), the EM approximate solution converges to the exact solution of (2.1) in the sense that

$$
\lim _{h \rightarrow 0} E\left[\sup _{0 \leq t \leq T}|y(t)-x(t)|^{2}\right]=0 .
$$




\section{A Motivating Example}

In the above section, we give the strong convergence numerical solution of SEPCAs under the local Lipschitz condition (H1) and the linear growth condition (H2). However, there are many SEPCAs that do not satisfy the linear growth condition, consider the following SEPCA:

$$
d x(t)=\left[-x^{3}(t)+x([t])\right] d t+\left[\sin x^{2}(t)+x([t])\right] d B(t) \quad \forall t \geq 0 .
$$

Clearly, the equation do not satisfy the linear growth condition (H2). But the example is analyzed under condition (H3) which covers many nonlinear SEPCAs. On the other hand, we have

$$
x\left(-x^{3}+y\right)+\frac{1}{2}\left(\sin x^{2}+y\right)^{2} \leq-x^{4}+x y+\left(\sin x^{2}\right)^{2}+y^{2} \leq 2\left(1+x^{2}+y^{2}\right) .
$$

In other words, the equation satisfies condition (H3). Moreover, we also have

$$
2 x^{T} f(x, y)+|g(x, y)|^{2} \leq|x|^{2}+2 K\left(1+|x|^{2}+|y|^{2}\right) \leq(1+2 K)\left(1+|x|^{2}+|y|^{2}\right) .
$$

We see clearly that (H3) follows from (H2). Therefore, The following result is more general than Theorem 3.2. Let us now turn to establish the convergence of the Euler-Maruyama method to (2.1) under the conditions (H1) and (H3).

\section{Convergence of the Euler-Maruyama Method under Monotone Condition}

In this section, we give the convergence of the EM method to (2.1) under the local Lipschitz condition (H1) and the monotone condition (H3). We prove the bounded $p$ th moment property of the EM approximate solution and the exact solution to (2.1) under the monotone condition (H3).

Lemma 6.1. Under the monotone condition (H3), there exists a positive constant $C_{5}$ such that the solution of (2.1) satisfies

$$
E \sup _{0 \leq t \leq T}|x(t)|^{p} \vee E \sup _{0 \leq t \leq T}|y(t)|^{p} \leq C_{5}
$$

where $C_{5}=C_{5}\left(p, T, K_{1}, x_{0}\right)$ is a constant independent of $h$. 
Proof. By Itô formula, for all $t \geq 0$, we have

$$
\begin{aligned}
&\left(1+|x(t)|^{2}\right)^{p / 2} \\
&=\left(1+|x(0)|^{2}\right)^{p / 2}+\int_{0}^{t} p\left(1+|x(t)|^{2}\right)^{(p-2) / 2} \\
& \quad \times\left[x(t)^{T} f(x(s), x([s]))+\frac{p-1}{2}|g(x(s), x([s]))|^{2}\right] d s \\
& \quad+\int_{0}^{t} p\left(1+|x(t)|^{2}\right)^{(p-2) / 2} x(s)^{T} g(x(s), x([s])) d B(s) \\
& \leq\left(1+|x(0)|^{2}\right)^{p / 2}+\int_{0}^{t} p\left(1+|x(t)|^{2}\right)^{(p-2) / 2}\left[K_{1}\left(1+|x(s)|^{2}+|x([s])|^{2}\right)\right] d s \\
&+\int_{0}^{t} p\left(1+|x(t)|^{2}\right)^{(p-2) / 2} x(s)^{T} g(x(s), x([s])) d B(s) .
\end{aligned}
$$

For any $t_{1} \in[0, T]$, we have

$$
\begin{aligned}
\sup _{0 \leq t \leq t_{1}}\left(1+|x(t)|^{2}\right)^{p / 2} & \\
\leq & \left(1+|x(0)|^{2}\right)^{p / 2}+E \sup _{0 \leq t \leq t_{1}} \int_{0}^{t} p\left(1+|x(t)|^{2}\right)^{(p-2) / 2}\left[K_{1}\left(1+|x(s)|^{2}+|x([s])|^{2}\right)\right] d s \\
& +E \sup _{0 \leq t \leq t_{1}}\left|\int_{0}^{t} p\left(1+|x(t)|^{2}\right)^{(p-2) / 2} x(s)^{T} g(x(s), x([s])) d B(s)\right| .
\end{aligned}
$$

By the Burkholder-Davis-Gundy inequality and Exercise 2.5 in [8], it is not difficult to show that

$$
\begin{aligned}
E \sup _{0 \leq t \leq t_{1}}\left|\int_{0}^{t} p\left(1+|x(t)|^{2}\right)^{(p-2) / 2} x(s)^{T} g(x(s), x([s])) d B(s)\right| \\
\quad \leq 3 E\left(\int_{0}^{t_{1}} p^{2}\left(1+|x(t)|^{2}\right)^{p-2}|x(s)|^{2}|g(x(s), x([s]))|^{2} d s\right)^{1 / 2} \\
\quad \leq 3 E\left(\sup _{0 \leq t \leq t_{1}}\left(1+|x(t)|^{2}\right)^{p / 2} \int_{0}^{t_{1}} p^{2}\left(1+|x(t)|^{2}\right)^{(p-2) / 2}|g(x(s), x([s]))|^{2} d s\right)^{1 / 2}
\end{aligned}
$$




$$
\begin{aligned}
& \leq 3 E\left(\sup _{0 \leq t \leq t_{1}}\left(1+|x(t)|^{2}\right)^{p / 2} \int_{0}^{t_{1}} p^{2} K_{1}\left(1+|x(t)|^{2}\right)^{(p-2) / 2}\left(1+|x(s)|^{2}+|x([s])|^{2}\right) d s\right)^{1 / 2} \\
& \leq 0.5 E \sup _{0 \leq t \leq t_{1}}\left(1+|x(t)|^{2}\right)^{p / 2}+4.5 E \int_{0}^{t_{1}} p^{2} K_{1}\left(1+|x(t)|^{2}\right)^{(p-2) / 2}\left(1+|x(s)|^{2}+|x([s])|^{2}\right) d s .
\end{aligned}
$$

Substituting (6.4) into (6.3) and using the Hölder inequality

$$
\begin{aligned}
\sup _{0 \leq t \leq t_{1}} & \left(1+|x(t)|^{2}\right)^{p / 2} \\
\leq & 2\left(1+|x(0)|^{2}\right)^{p / 2}+2 p K_{1}(1+4.5 p) E \int_{0}^{t_{1}}\left(1+|x(t)|^{2}\right)^{(p-2) / 2}\left(1+|x(s)|^{2}+|x([s])|^{2}\right) d s \\
\leq & 2\left(1+|x(0)|^{2}\right)^{p / 2} \\
& +2 p K_{1}(1+4.5 p)\left(E \int_{0}^{t_{1}}\left(1+\left.x(t)\right|^{2}\right)^{p / 2} d s\right)^{(p-2) / p} \\
& \times\left(E \int_{0}^{t_{1}}\left(1+|x(s)|^{2}+|x([s])|^{2}\right)^{p / 2} d s\right)^{2 / p} \\
\leq & 2\left(1+|x(0)|^{2}\right)^{p / 2} \\
& +2 p K_{1}(1+4.5 p)\left(T E \sup _{0 \leq t \leq t_{1}}\left(1+|x(t)|^{2}\right)^{p / 2}\right)^{(p-2) / p} \\
& \times\left(E \int_{0}^{t_{1}}\left(1+|x(s)|^{2}+|x([s])|^{2}\right)^{p / 2} d s\right)^{2 / p} \cdot \\
& (1+6.5)
\end{aligned}
$$

\section{So we obtain}

$$
\begin{aligned}
E \sup _{0 \leq t \leq t_{1}}\left(1+|x(t)|^{2}\right)^{p / 2} & \\
\leq & {\left[2\left(1+|x(0)|^{2}\right)^{p / 2}+2 p K_{1}(1+4.5 p) T^{(p-2) / p}\left(E \int_{0}^{t_{1}}\left(1+|x(s)|^{2}+|x([s])|^{2}\right)^{p / 2} d s\right)^{2 / p}\right]^{p / 2} } \\
\leq & 2^{(p / 2)-1}\left[2\left(1+|x(0)|^{2}\right)^{p / 2}\right]^{p / 2} \\
& +2^{(p / 2)-1}\left[2 p K_{1}(1+4.5 p) T^{(p-2) / p}\left(E \int_{0}^{t_{1}}\left(1+|x(s)|^{2}+|x([s])|^{2}\right)^{p / 2} d s\right)^{2 / p}\right]^{p / 2}
\end{aligned}
$$




$$
\begin{aligned}
& \leq 2^{p-1}\left(1+|x(0)|^{2}\right)^{p^{2} / 4}+2^{p-1}\left[p K_{1}(1+4.5 p) T^{(p-2) / p}\right]^{p / 2} E \int_{0}^{t_{1}}\left(1+|x(s)|^{2}+|x([s])|^{2}\right)^{p / 2} d s \\
& \leq 2^{p-1}\left(1+|x(0)|^{2}\right)^{p^{2} / 4}+2^{(3 p / 2)-1}\left[p K_{1}(1+4.5 p) T^{(p-2) / p}\right]^{p / 2} \int_{0}^{t_{1}} E \sup _{0 \leq u \leq s}\left(1+|x(u)|^{2}\right)^{p / 2} d s .
\end{aligned}
$$

By the Gronwall inequality, we must get

$$
E \sup _{0 \leq t \leq T}\left(|x(t)|^{p}\right) \leq C_{5}
$$

where $C_{5}=C_{5}\left(p, T, K, x_{0}\right)$ is a constant independent of $h$. Similarly, we can show that

$$
\sup _{0 \leq t \leq T}\left(|y(t)|^{p}\right) \leq C_{5}
$$

The proof is completed.

According to Theorem 3.2, we obtain the following.

Theorem 6.2. Under the conditions (H1) and (H3), the EM approximate solution converges to the exact solution of (2.1) in the sense that

$$
\lim _{h \rightarrow 0} E\left(\sup _{0 \leq t \leq T}|y(t)-x(t)|^{2}\right)=0
$$

\section{Acknowledgments}

The financial support from the National Natural Science Foundation of China (no. 11071050) and the Youths' Key Projects of Heilongjiang Provincial Education Department of P. R. China (no. 1155G001) is gratefully acknowledged.

\section{References}

[1] K. L. Cooke and J. Wiener, "Retarded differential equations with piecewise constant delays," Journal of Mathematical Analysis and Applications, vol. 99, no. 1, pp. 265-297, 1984.

[2] J. Wiener, Generalized Solutions of Functional-Differential Equations, World Scientific Publishing, River Edge, NJ, USA, 1993.

[3] M. H. Song, Z. W. Yang, and M. Z. Liu, "Stability of $\theta$-methods for advanced differential equations with piecewise continuous arguments," Computers $\mathcal{E}$ Mathematics with Applications, vol. 49, no. 9-10, pp. 1295-1301, 2005.

[4] A. Friedman, Stochastic Differential Equations and Applications, vol. 1, Academic Press, New York, NY, USA, 1975.

[5] X. Mao, Stochastic Differential Equations and Applications, Horwood Publishing, Chichester, UK, 2nd edition, 1997.

[6] X. Mao, "Numerical solutions of stochastic functional differential equations," LMS Journal of Computation and Mathematics, vol. 6, pp. 141-161, 2003. 
[7] X. Mao and S. Sabanis, "Numerical solutions of stochastic differential delay equations under local Lipschitz condition," Journal of Computational and Applied Mathematics, vol. 151, no. 1, pp. 215-227, 2003.

[8] X. Mao and C. Yuan, Stochastic Differential Equations with Markovian Switching, Imperial College Press, London, UK, 2006.

[9] H. Y. Dai and M. Z. Liu, "Mean square stability of stochastic differential equations with piecewise continuous arguments," Journal of Natural Science of Heilongjiang University, vol. 25, no. 5, pp. 625-629, 2008.

[10] C. T. Ng and W. Zhang, "Invariant curves for planar mappings," Journal of Difference Equations and Applications, vol. 3, no. 2, pp. 147-168, 1997. 


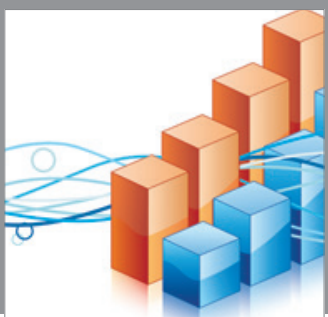

Advances in

Operations Research

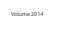

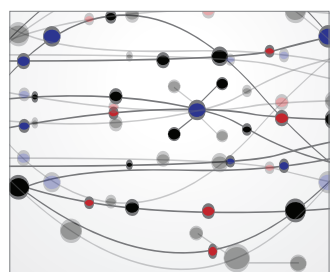

\section{The Scientific} World Journal
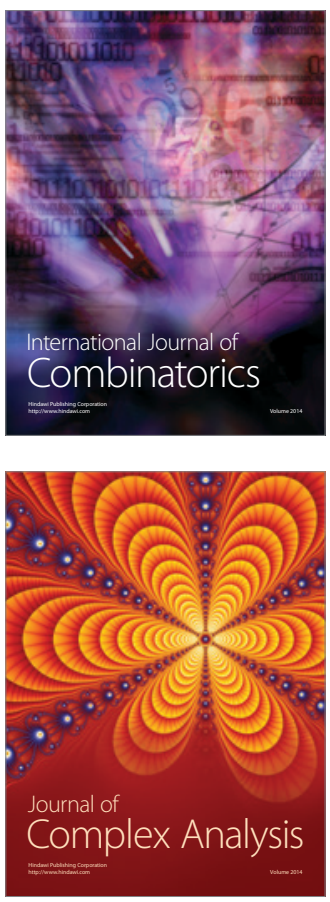

International Journal of

Mathematics and

Mathematical

Sciences
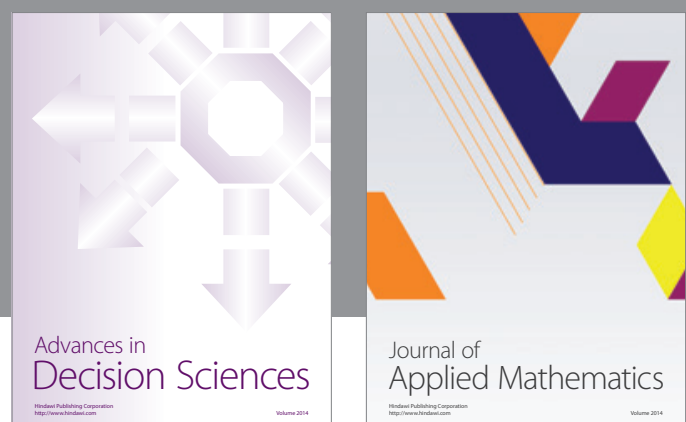

Journal of

Applied Mathematics
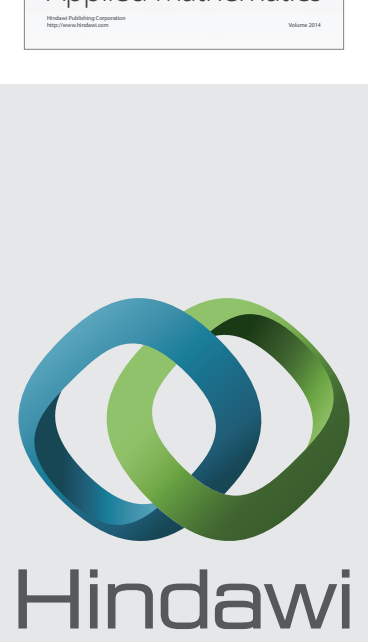

Submit your manuscripts at http://www.hindawi.com
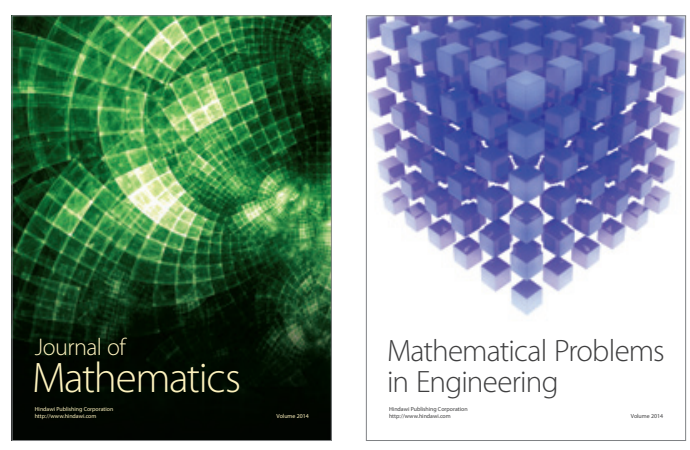

Mathematical Problems in Engineering
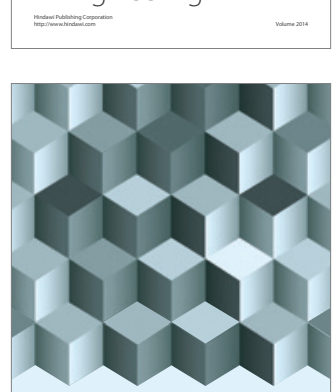

Journal of

Function Spaces
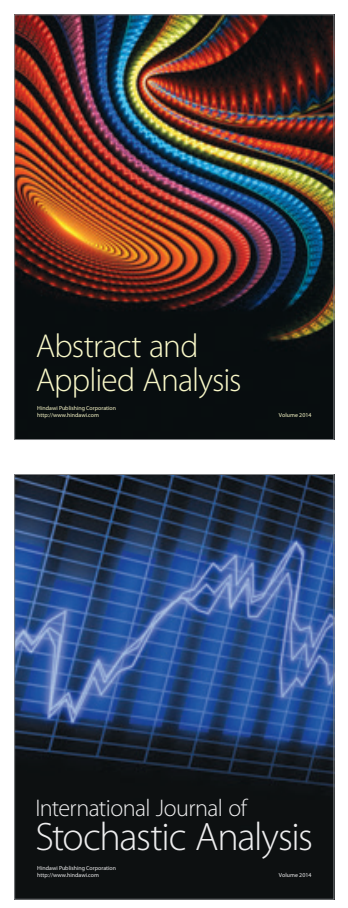

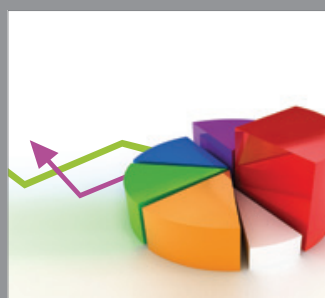

ournal of

Probability and Statistics

Promensencen
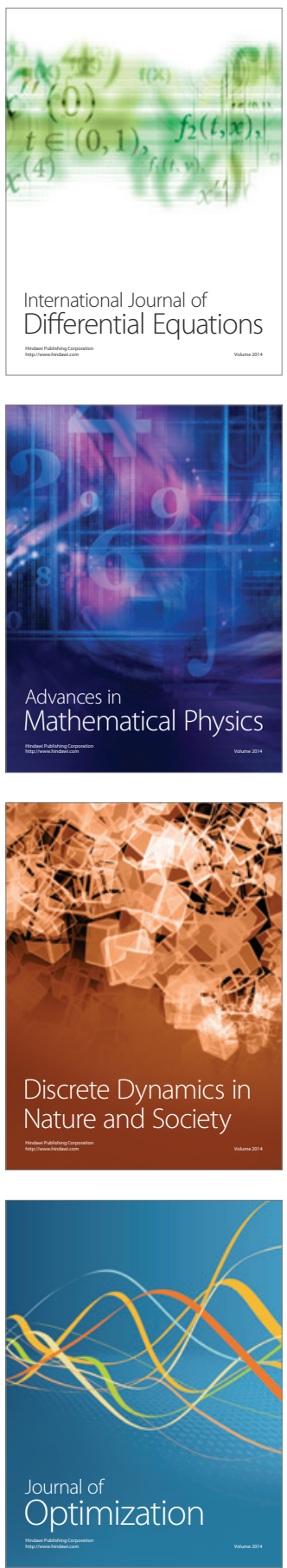